\title{
An Approach to Robust Control of the Hopf Bifurcation
}

\author{
Giacomo Innocenti, ${ }^{1,}{ }^{2}$ Roberto Genesio, ${ }^{1}$ and Alberto Tesi ${ }^{1}$ \\ ${ }^{1}$ Dipartimento di Sistemi e Informatica, Università di Firenze, Via S. Marta 3, 50135 Firenze, Italy \\ ${ }^{2}$ Dipartimento di Ingegneria dell'Informazione, Università di Siena, Via Roma 56, 53100 Siena, Italy \\ Correspondence should be addressed to Giacomo Innocenti, giacomo.innocenti@gmail.com
}

Received 1 December 2010; Accepted 12 January 2011

Academic Editor: F. Marcellan

Copyright (c) 2011 Giacomo Innocenti et al. This is an open access article distributed under the Creative Commons Attribution License, which permits unrestricted use, distribution, and reproduction in any medium, provided the original work is properly cited.

The paper illustrates a novel approach to modify the Hopf bifurcation nature via a nonlinear state feedback control, which leaves the equilibrium properties unchanged. This result is achieved by recurring to linear and nonlinear transformations, which lead the system to locally assume the ordinary differential equation representation. Third-order models are considered, since they can be seen as proper representatives of a larger class of systems. The explicit relationship between the control input and the Hopf bifurcation nature is obtained via a frequency approach, that does not need the computation of the center manifold.

\section{Introduction}

In many fields of science, spacing from physics to biology and from engineering to economics, the learning of phenomena, characterized by complex dynamics, and their properties opened several new opportunities in the management of real processes. Consequently, new problems arose in the area of control system design and among these particular attention has been devoted to what is called the "bifurcation control".

A nonlinear dynamical system frequently presents variations of its parameters, and this can happen because of actual slow structural modifications of the considered phenomenon or due to the uncertainties affecting its modeling. When such variations happen around certain critical conditions, the system exhibits a qualitative change of behaviour defined as a bifurcation. Since the related transitions can lead to substantial effects, even destructive, for the system in study, their control has been deeply considered through more than two decades, covering theoretical aspects and various concrete applications. In this perspective, the control objective is to modify an existing bifurcation, changing its characteristics or suitably moving it or introducing a new one, in order to avoid dangerous conditions and obtain a desirable behaviour (see $[1,2]$ for an overview). 
In particular, the important case of Hopf bifurcation, leading to system oscillations, has been studied by different approaches, usually reducing the system to the essential secondorder dynamics, that is, that concerning its central manifold. Time domain procedures use washout filters to stabilize dynamical feedback in $[3,4]$, while linear and nonlinear state feedback are considered in [5-7] and refer particularly to controller normal forms in [812]. Frequency domain techniques have also been studied in terms of Harmonic Balance, as reported in [13-15].

This paper exploits linear and local nonlinear transformations, derived from controller normal form theory, in order to rewrite the original state equations of the system as a scalar ordinary differential equation. Then, the use of a frequency approach based on the harmonic balance method leads to analytically relate the original input to the bifurcation parameters, so giving a mathematical tool to directly design the control input once the system is given. Conditions to provide a robust control are investigated and discussed, as well. In particular, third-order systems are here considered and sufficient conditions are provided to obtain the corresponding scalar ODE representation. It is worth observing that such reduced models frequently give an efficient representation of many processes, since they can exhibit a large variety of complex dynamics (see, e.g., [16] and references within). This is the case of systems characterized by fast third-order subdynamics, where the higher order components can be seen as a slow varying external unknown input. Moreover, the scalar differential equation form is an essential point to directly derive and approximate the system dynamics around the Hopf bifurcation, since it allows one to detect the nature of such a phenomenon without recurring to the center manifold. Finally, the main idea of the paper appears in principle to be suitable for an extension to higher order systems.

The paper is organized as follows. In Section 2, we address the considered framework. Then, we derive in Section 3 a set of linear and nonlinear transformations, which lead the system to locally assume the ODE representation. An illustrative example is presented in Section 4 and some considerations are reported in Section 5. A mathematical appendix ends the paper.

\section{Problem Formulation}

Let us consider the affine control system

$$
\dot{\xi}=F(\xi ; \mu)+G(\xi ; \mu) u
$$

where

$$
\begin{gathered}
\xi \in \mathbb{R}^{3}, \\
u \in \mathbb{R}, \\
\mu \in \Gamma \subset \mathbb{R}^{n}, \\
F(\xi ; \mu): \mathbb{R}^{3} \times \mathbb{R}^{n} \longrightarrow \mathbb{R}^{3}, \\
G(\xi ; \mu): \mathbb{R}^{3} \times \mathbb{R}^{n} \longrightarrow \mathbb{R}^{3} .
\end{gathered}
$$


Assume that $\mu$ is a parametric vector, which may change in time according to an unknown or unmodeled slow dynamics, so that the related modifications can be considered a quasistatic process. Without loss of generality, let $\xi_{e}(\mu)$ represent the equilibrium point of the autonomous part of system (2.1), that is, the point such that

$$
F\left(\xi_{e}(\mu) ; \mu\right)=0_{3}, \quad \forall \mu \in \Gamma,
$$

and assume that $\xi_{e}(\mu)$ undergoes a Hopf bifurcation at $\mu=\mu_{0} \in \Gamma$.

In the following, we develop an analytic method to modify the nature of such bifurcation via a locally nonlinear input, that leaves unaltered the fixed points manifold and their stability properties.

Then, let us perform the change of coordinate, that brings the equilibrium points manifold (2.3) in the origin, that is, define $x=\xi-\xi_{e}(\mu)$, so that

$$
\begin{aligned}
\dot{x} & =\dot{\xi} \\
& =F\left(x+\xi_{e}(\mu) ; \mu\right)+G\left(x+\xi_{e}(\mu) ; \mu\right) w \\
& =F\left(\xi_{e}(\mu) ; \mu\right)+\frac{\partial F}{\partial \xi}\left(\xi_{e}(\mu) ; \mu\right) x+\cdots+\left(G\left(\xi_{e}(\mu) ; \mu\right)+\frac{\partial G}{\partial \xi}\left(\xi_{e}(\mu) ; \mu\right) x+\cdots\right) w \\
& =\frac{\partial F}{\partial \xi}\left(\xi_{e}(\mu) ; \mu\right) x+\cdots+\left(G\left(\xi_{e}(\mu) ; \mu\right)+\frac{\partial G}{\partial \xi}\left(\xi_{e}(\mu) ; \mu\right) x+\cdots\right) w .
\end{aligned}
$$

By denoting

$$
\begin{gathered}
\tilde{f}(x ; \mu)=F\left(x+\xi_{e}(\mu) ; \mu\right)-F\left(\xi_{e}(\mu) ; \mu\right), \\
\tilde{A}(\mu), \quad \text { such that } \tilde{A}(\mu) x=\tilde{f}^{[1]}(x ; \mu), \\
\tilde{g}(x ; \mu)=G\left(x+\xi_{e}(\mu) ; \mu\right), \\
\tilde{B}(\mu)=G\left(\xi_{e}(\mu) ; \mu\right),
\end{gathered}
$$

we obtain the transformed system

$$
\dot{x}=\tilde{A}(\mu) x+\tilde{f}^{[2]}(x ; \mu)+\cdots+\left(\widetilde{B}(\mu)+\tilde{g}^{[1]}(x ; \mu)+\cdots\right) w .
$$

Since it is wellknown that, to study the super or subcritical nature of a nondegenerate Hopf bifurcation, it is sufficient to consider the quadratic and cubic local terms [17-20], without loss of generality in the following we will focus only on the subsystem

$$
\dot{x}=\tilde{A}(\mu) x+\tilde{f}^{[2]}(x ; \mu)+\tilde{f}^{[3]}(x ; \mu)+\left(\widetilde{B}(\mu)+\tilde{g}^{[1]}(x ; \mu)+\tilde{g}^{[2]}(x ; \mu)\right) w .
$$

In the next section, we narrow our theory to linearly controllable affine systems, that is, models whose linear components satisfy the controllability condition (see, e.g., [21]). 


\section{Hopf Bifurcation in Local ODE Models}

Hereafter, we analytically point out a class of third-order affine control systems, which can be locally represented via ODE models. The corresponding conditions are only sufficient and, thus, the proposed method could be generalized to a large variety of systems though this would require an increased amount of computations. However, a byproduct of our theory is the explicit relationship between the coefficients of the starting model and its local ODE representation. Hence, once such a transformation has been computed, it can be used as a look-up table for every other system in that class.

Then, let us focus on linearly controllable systems. The following result holds.

Theorem 3.1. Consider system (2.7) and assume that $(\widetilde{A}(\mu), \widetilde{B}(\mu))$ is a controllable pair for each $\mu \in \Gamma$, then, an invertible $T(\mu) \in \mathbb{R}^{3 \times 3}$ and

$$
v(\mu)=\left[\begin{array}{lll}
v_{1}(\mu) & v_{2}(\mu) & v_{3}(\mu)
\end{array}\right]
$$

exist such that the linear transformation

$$
\begin{gathered}
y=T(\mu) x, \\
u=v(\mu) T(\mu) x+w
\end{gathered}
$$

modifies the system into the model

$$
\dot{y}=A y+f^{[2]}(y ; \mu)+f^{[3]}(y ; \mu)+\left(B+g^{[1]}(y ; \mu)+g^{[2]}(y ; \mu)\right) u,
$$

where the pair $(A, B)$ is in Brunovsky form, that is,

$$
A=\left[\begin{array}{lll}
0 & 1 & 0 \\
0 & 0 & 1 \\
0 & 0 & 0
\end{array}\right], \quad B=\left[\begin{array}{l}
0 \\
0 \\
1
\end{array}\right]
$$

It is worth underlining that transformation (3.2) can be directly computed from (A.2) via standard algorithms used to point out the proper $T(\mu)$ satisfying (A.1) (see the appendix).

Corollary 3.2. System (3.3) controlled via the input

$$
u=v(\mu) y+k^{[2]}(y ; \mu)+k^{[3]}(y ; \mu)
$$

and system (2.7) with

$$
w=k^{[2]}(T(\mu) x ; \mu)+k^{[3]}(T(\mu) x ; \mu)
$$

are locally equivalent at their equilibria $\xi_{e}(\mu) \leftrightarrow 0_{3}$. 
Hereafter, we derive a class of affine control systems, which can be locally described as ODEs, when their inputs are designed as a state feedback. This approach is motivated by the existence of simple though powerful analytic mathematical tools, developed to study Hopf bifurcations in third order nonlinear ODE systems [19, 20, 22].

Theorem 3.3. Consider the linearly controllable system (3.3), with $(A, B)$ in Brunovsky form, then, by means of a nonlinear transformation

$$
\begin{gathered}
y=z+\varphi^{[2]}(z ; \mu)+\varphi^{[3]}(z ; \mu), \\
v=u+\psi^{[2]}(z, u ; \mu)+\psi^{[3]}(z, u ; \mu),
\end{gathered}
$$

it assumes the form

$$
\begin{gathered}
\dot{z}_{1}=z_{2}+\gamma_{16}(\mu) z_{3}^{2}+\left(\delta_{17}(\mu)\right. \\
\left.z_{1}+\delta_{18}(\mu) z_{2}+\delta_{19}(\mu) z_{3}\right) z_{3}^{2} \\
\dot{z}_{2}=z_{3} \\
\dot{z}_{3}=v
\end{gathered}
$$

which is locally equivalent to (3.3) around the equilibrium in the origin.

Corollary 3.4. Consider system (3.9), where the parameters $\gamma_{16}(\mu), \delta_{17}(\mu), \delta_{18}(\mu)$, and $\delta_{19}(\mu)$ are computed from the coefficients of the polynomial terms of the nonlinearities of model (3.3) according to Tables 1, 2, and 3. If the condition

$$
\gamma_{16}(\mu)=\delta_{17}(\mu)=\delta_{18}(\mu)=\delta_{19}(\mu)=0, \quad \forall \mu \in \Gamma
$$

holds, then (3.9) can be transformed into the ODE model

$$
\dddot{\zeta}=v,
$$

via the linear change of coordinates

$$
z_{1}=\zeta, \quad z_{2}=\dot{\zeta}, \quad z_{3}=\ddot{\zeta} .
$$

The systems satisfying condition (3.9) can be explicitly pointed out by means of the parametric relationships reported in the tables. However, they can also be represented via the functional characterization of the following statement. 
Table 1: Relationships between the coefficients of $f^{[2]}(z), g^{[1]}(z)$, and $\varphi^{[2]}(z)$ in (A.10)-(A.12), that is, pertaining the quadratic part of the nonlinear transformation (3.7).

\begin{tabular}{c}
$\alpha_{11}$ can be freely assigned. \\
$\alpha_{12}=a_{13}+\alpha_{23}=a_{13}+b_{21}$ \\
$\alpha_{13}=b_{11}$ \\
$\alpha_{14}=\frac{1}{2}\left(a_{15}+\alpha_{25}-b_{11}\right)=\frac{1}{2}\left(a_{15}+b_{22}-b_{11}\right)$ \\
$\alpha_{15}=b_{12}$ \\
$\alpha_{16}=\frac{1}{2} b_{13}$ \\
$\alpha_{21}=-a_{21}$ \\
$\alpha_{22}=-a_{21}+2 \alpha_{11}$ \\
$\alpha_{23}=b_{21}$ \\
$\gamma_{16}=\alpha_{26}+a_{16}-b_{12}=\frac{1}{2} b_{23}+a_{16}-b_{12}$ \\
$\alpha_{24}=-a_{14}+\alpha_{12}=-a_{14}+a_{13}+b_{21}$ \\
$\alpha_{25}=b_{22}$ \\
$\alpha_{26}=\frac{1}{2} b_{23}$ \\
$\alpha_{34}=-a_{24}+a_{22}=-a_{21}$ \\
$\alpha_{32}=-a_{22}+2 \alpha_{21}=-a_{22}+2\left(-a_{21}\right)$ \\
\hline$\alpha_{23}=-a_{23}+\left(-a_{21}+2 \alpha_{11}\right)$ \\
\end{tabular}

Theorem 3.5. A system of the form (3.9) satisfies condition (3.10) if and only if $\varphi^{[2]}(z), \varphi^{[3]}(z)$ : $\mathbb{R}^{3} \rightarrow \mathbb{R}^{3}$ and $\psi_{z}^{[2]}(z), \psi_{z}^{[3]}(z), \psi_{u}^{[1]}(z), \psi_{u}^{[2]}(z): \mathbb{R}^{3} \rightarrow \mathbb{R}$ exist, which satisfy

$$
\begin{gathered}
A \varphi^{[2]}(z)+f^{[2]}(z)-\frac{\partial \varphi^{[2]}}{\partial z} A z=B \psi_{z}^{[2]}(z), \\
g^{[1]}(z)-\frac{\partial \varphi^{[2]}}{\partial z} B=B \varphi_{u}^{[1]}(z), \\
A \varphi^{[3]}(z)+f^{[3]}(z)+\frac{\partial f^{[2]}}{\partial z} \varphi^{[2]}(z)-\frac{\partial \varphi^{[2]}}{\partial z} B \varphi_{z}^{[2]}(z)-\frac{\partial \varphi^{[3]}}{\partial z} A z=B \psi_{z}^{[3]}(z), \\
g^{[2]}(z)+\frac{\partial g^{[1]}}{\partial z} \varphi^{[2]}(z)-\frac{\partial \varphi^{[2]}}{\partial z} B \psi_{u}^{[1]}(z)-\frac{\partial \varphi^{[3]}}{\partial z} B=B \varphi_{u}^{[2]}(z) .
\end{gathered}
$$


Table 2: Relationships between the coefficients of $\hat{f}^{[3]}(z)$ and those of $f^{[3]}(z), f^{[2]}(z)$ and $\varphi^{[2]}(z)$ as they result from condition (A.19).

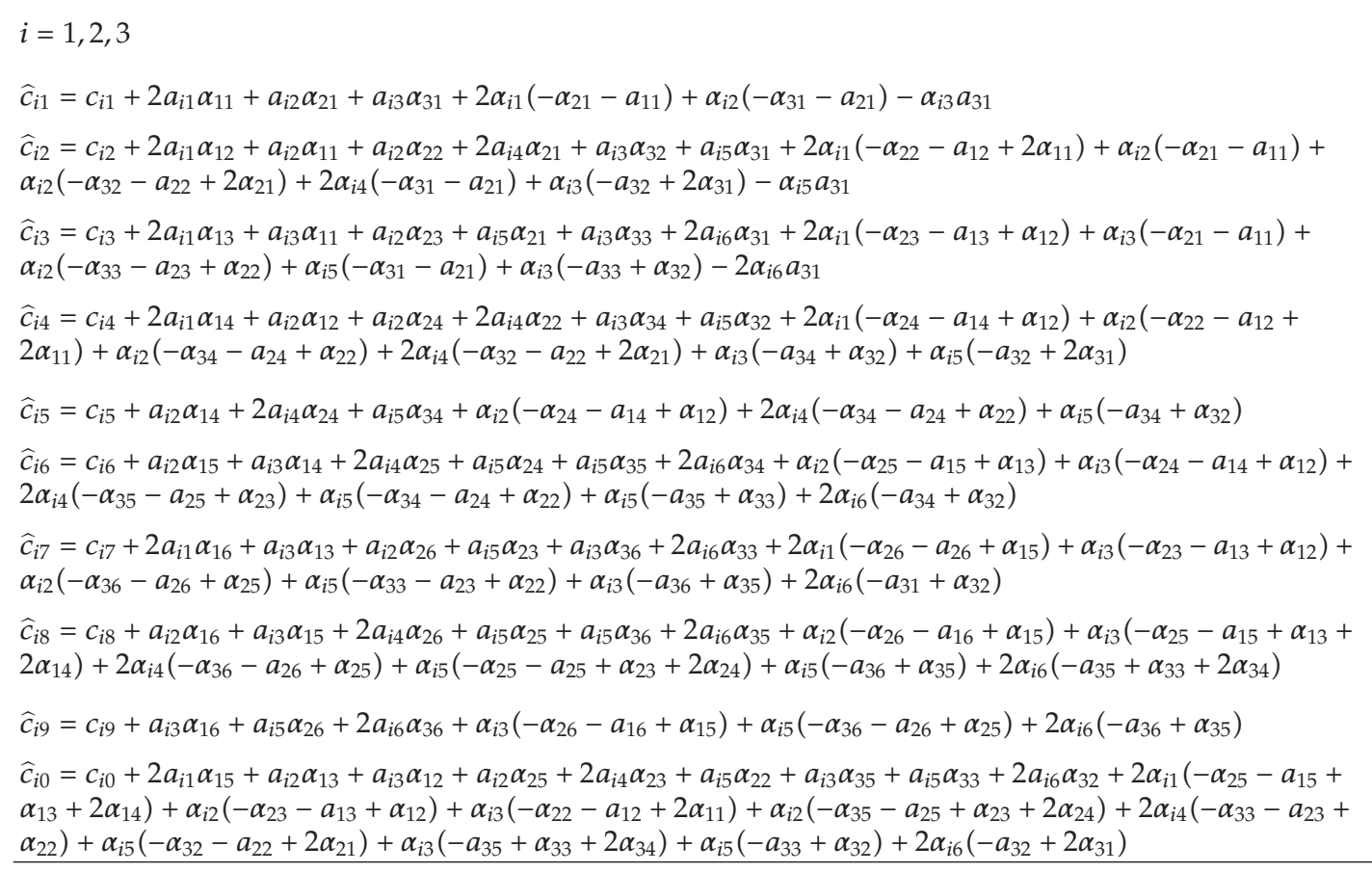

Table 3: Relationships between the coefficients of $g^{[1]}(z)$ and those of $\hat{g}^{[2]}(z)$ and $\varphi^{[2]}(z)$ as they result from conditions (A.20).

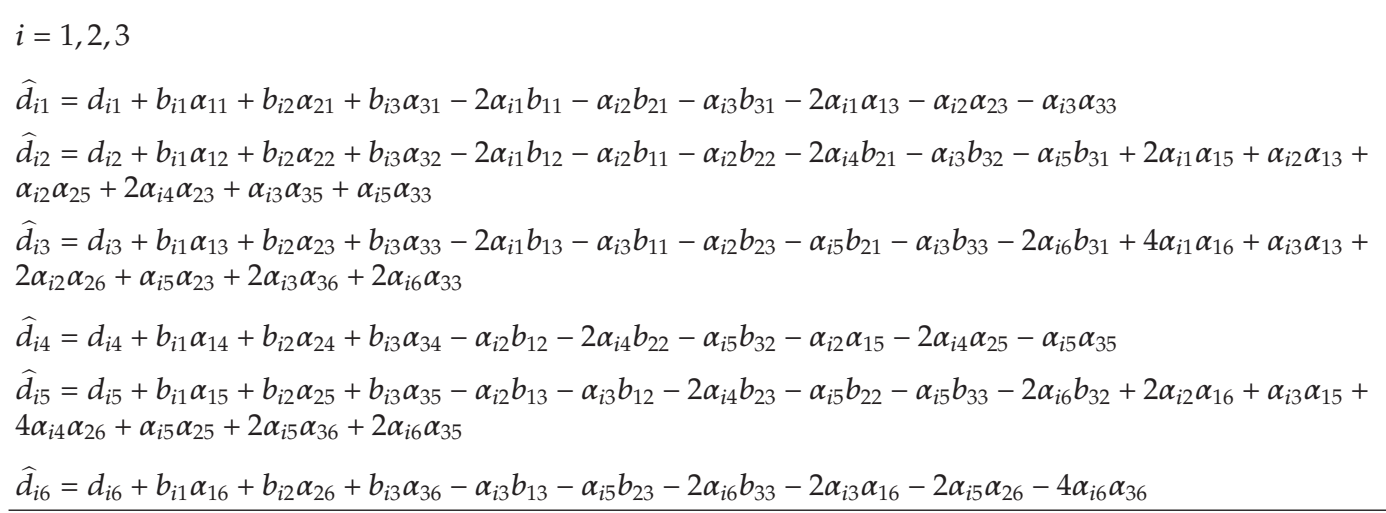

Hereafter, a local nonlinear transformation, which maintains unaltered the linear part of the system, is introduced.

Theorem 3.6. System (3.3) controlled via the input

$$
u=v(\mu) y+k^{[2]}(y ; \mu)+k^{[3]}(y ; \mu)
$$


and system (3.9), or the ODE model (3.11), controlled by

$$
v=v(\mu) z+v^{[2]}(z ; \mu)+v^{[3]}(z ; \mu)
$$

are locally equivalent around their equilibria in the origin, when the following conditions, related also to the polynomial functions in (3.7) and (3.8), are satisfied:

$$
\begin{aligned}
k^{[2]}(z ; \mu)= & v^{[2]}(z ; \mu)-v(\mu) \varphi^{[2]}(z ; \mu)-\psi^{[2]}(z, v(\mu) z ; \mu), \\
k^{[3]}(z ; \mu)= & v^{[3]}(z ; \mu)-v(\mu) \varphi^{[3]}(z, v(\mu) z ; \mu)-\frac{\partial k^{[2]}}{\partial z} \varphi^{[2]}(z ; \mu) \\
& -\frac{\partial \psi^{[2]}}{\partial u} \varphi^{[2]}(z ; \mu)-\frac{\partial \psi^{[2]}}{\partial u} k^{[2]}(z ; \mu)-\psi^{[3]}(z, v(\mu) z ; \mu) .
\end{aligned}
$$

The above Theorem 3.6 allows us to design the nonlinear terms of the control input of system (3.3) and, then, of the original model (2.7), just by referring to the ODE system (3.11) driven by the input

$$
v=v_{1}(\mu) \zeta+v_{2}(\mu) \dot{\zeta}+v_{3}(\mu) \ddot{\zeta}+v^{[2]}(\zeta, \dot{\zeta}, \ddot{\zeta} ; \mu)+v^{[3]}(\zeta, \dot{\zeta}, \ddot{\zeta} ; \mu) .
$$

Therefore, the Hopf bifurcation nature can be modified by a suitable choice of the nonlinearities $v^{[2]}(\zeta, \dot{\zeta}, \ddot{\zeta} ; \mu)$ and $v^{[3]}(\zeta, \dot{\zeta}, \ddot{\zeta} ; \mu)$. Since the control system (3.11)-(3.20) is an ODE model, a generalized frequency approach, as that shown in [20, 22], can still be fruitfully employed. Moreover, by computing also the explicit relationship between the coefficients of the nonlinearities in the starting model and in the final ODE system, one can also point out the simplest input $w$, that provides the desired result. However, in order to illustrate this frequency approach by keeping the computational burden low, hereafter we describe how the bifurcation is affected by the only quadratic term $v^{[2]}(\zeta, \dot{\zeta}, \ddot{\zeta} ; \mu)$, when the cubic component $v^{[3]}(\zeta, \dot{\zeta}, \ddot{\zeta} ; \mu)$ is set to zero.

Proposition 3.7. Consider the ODE system

$$
\begin{aligned}
\dddot{\zeta}= & v_{3}(\mu) \ddot{\zeta}+v_{2}(\mu) \dot{\zeta}+v_{1}(\mu) \zeta \\
& +r_{1}(\mu) \zeta^{2}+r_{2}(\mu) \dot{\zeta} \zeta+r_{3} \ddot{\zeta} \zeta+r_{4}(\mu) \dot{\zeta}^{2}+r_{5}(\mu) \ddot{\zeta} \dot{\xi}+r_{6}(\mu) \ddot{\zeta}^{2}=0,
\end{aligned}
$$

and assume that its equilibrium point in $\zeta=0$ undergoes a nondegenerate Hopf bifurcation at $\mu=\mu_{0}$. Then the bifurcation is supercritical, if $\operatorname{det} M\left(\mu_{0}\right)<0$, and subcritical if $\operatorname{det} M\left(\mu_{0}\right)>0$, where matrix 
$M(\mu)$ is defined as

$$
M=\left[\begin{array}{ccccc}
-v_{3} & \frac{1}{2}\left(-r_{1}-\left(-r_{4}+r_{3}\right) v_{2}-r_{6} v_{2}^{2}\right) & 0 & 0 & 0 \\
-2 r_{1}-r_{3} v_{2} & 0 & A & B & 2 v_{3}\left(-v_{2}\right)^{1 / 2} \\
r_{2}\left(-v_{2}\right)^{1 / 2} & 0 & B & A & -2 v_{2} \\
0 & \frac{1}{2}\left(-r_{1}+\left(-r_{3}-r_{4}\right) v_{2}-r_{6} v_{2}^{2}\right) & 3 v_{1} & -6\left(-v_{2}\right)^{3 / 2} & 0 \\
0 & \frac{1}{2}\left(-v_{2}\right)^{1 / 2}\left(r_{2}+r_{5} v_{2}\right) & 6\left(-v_{2}\right)^{3 / 2} & 3 v_{1} & 0
\end{array}\right],
$$

where $A$ denotes $-r_{1}-\left(-2 r_{4}+(5 / 2) r_{3}\right) v_{2}-4 r_{6} v_{2}^{2}$ and $\boldsymbol{B}$ denotes $\left(-v_{2}\right)^{1 / 2}\left((-1 / 2) r_{2}+r_{5} v_{2}\right)$, being the dependency on $\mu$ neglected for the sake of simplicity (for a detailed proof of the statement refer to [19]).

Remark 3.8. It is worth noticing that also the limit cycle features can be set up via the same frequency approach, as illustrated in $[19,20]$.

Proposition 3.7, along with the explicit relationships of Tables 1-4 between the coefficients of the starting systems and the final ODE model, can be fruitfully exploited to design the nonlinear components of input $w$ in order to obtain the desired results about the Hopf bifurcation at $\mu=\mu_{0}$.

To this aim, it is important to underline that in general the control input designed this way depends on the bifurcation parameter $\mu$. However, in certain situations the parameters of the model, which slowly vary in time according to an unknown law, could not be accessible to compute the proper input. In such a case, where $\mu$ cannot be observed, control $w$ solves the problem only if it does not depend on it. This scenario can be regarded as a robust bifurcation control approach. Such a case is summarized by the following statement.

Proposition 3.9 (Robust control design). Let one consider system (2.7) along with the control input (3.6), computed according to (3.19) and related conditions. Set

$$
\begin{gathered}
v^{[2]}(z ; \mu)=r_{1}(\mu) z_{1}^{2}+r_{2}(\mu) z_{2} z_{1}+r_{3}(\mu) z_{3} z_{1}+r_{4}(\mu) z_{2}^{2}+r_{5}(\mu) z_{3} z_{2}+r_{6}(\mu) z_{3}^{2} \\
v^{[3]}(z ; \mu)=0
\end{gathered}
$$

and denote by

$$
r(\mu)=\left[\begin{array}{llllll}
r_{1}(\mu) & r_{2}(\mu) & r_{3}(\mu) & r_{4}(\mu) & r_{5}(\mu) & r_{6}(\mu)
\end{array}\right]^{T}
$$

the control parametric vector. Moreover, consider matrix (3.22) and define the sets

$$
\begin{aligned}
& R_{\text {sup }}=\left\{r(\mu): \operatorname{det} M\left(\mu_{0}\right)<0\right\}, \\
& R_{\text {sup }}=\left\{r(\mu): \operatorname{det} M\left(\mu_{0}\right)>0\right\},
\end{aligned}
$$


Table 4: Relationships between the coefficients of $f^{[3]}(z), g^{[2]}(z)$, and $\varphi^{[3]}(z)$ in (A.10)-(A.12), that is, pertaining the cubic part of the nonlinear transformation (3.7).

\begin{tabular}{|c|}
\hline $\begin{array}{c}\beta_{11} \text { can be freely assigned. } \\
\beta_{12}=\beta_{23}+\widehat{c}_{13}=\widehat{d}_{21}+\widehat{c}_{13} \\
\beta_{13}=\widehat{d}_{11} \\
\beta_{14}=\frac{1}{2}\left(\beta_{20}+\widehat{c}_{10}-2 \beta_{13}\right)=\frac{1}{2}\left(\widehat{d}_{22}+\widehat{c}_{10}-2 \widehat{d}_{11}\right) \\
\beta_{15}=\frac{1}{3}\left(\beta_{26}+\widehat{c}_{16}-\beta_{10}\right)=\frac{1}{3}\left(\widehat{d}_{24}+\widehat{c}_{16}-\widehat{d}_{12}\right) \\
\beta_{16}=\widehat{d}_{14} \\
\beta_{17}=\frac{1}{2} \widehat{d}_{13} \\
\beta_{18}=\frac{1}{2} \widehat{d}_{15} \\
\beta_{19}=\frac{1}{3} \widehat{d}_{16} \\
\beta_{10}=\widehat{d}_{12}\end{array}$ \\
\hline $\begin{array}{c}\beta_{21}=-\widehat{c}_{11} \\
\beta_{22}=-\widehat{c}_{12}+3 \beta_{11}=-\widehat{c}_{12}+3 \beta_{11} \\
\beta_{23}=\widehat{d}_{21} \\
\beta_{24}=-\widehat{c}_{14}+2 \beta_{12}=-\widehat{c}_{14}+2\left(\widehat{d}_{21}+\widehat{c}_{13}\right) \\
\beta_{25}=-\widehat{c}_{15}+\beta_{14}=-\widehat{c}_{15}+\frac{1}{2}\left(\widehat{d}_{22}+\widehat{c}_{10}-2 \widehat{d}_{11}\right) \\
\beta_{26}=\widehat{d}_{24} \\
\beta_{27}=\frac{1}{2} \widehat{d}_{23} \\
\beta_{28}=\frac{1}{2} \widehat{d}_{25} \\
\beta_{29}=\frac{1}{3} \widehat{d}_{26} \\
\beta_{20}=\widehat{d}_{22} \\
\beta_{31}=-\widehat{c}_{21}\end{array}$ \\
\hline $\begin{array}{c}\beta_{32}=-\widehat{c}_{22}+3 \beta_{21}=-\widehat{c}_{22}-3 \widehat{c}_{11} \\
\beta_{33}=-\widehat{c}_{23}+\beta_{22}=-\widehat{c}_{23}-\widehat{c}_{12}+3 \beta_{11} \\
\beta_{34}=-\widehat{c}_{24}+2 \beta_{22}=-\widehat{c}_{24}+2\left(-\widehat{c}_{12}+3 \beta_{11}\right) \\
\beta_{35}=-\widehat{c}_{25}+\beta_{24}=-\widehat{c}_{25}-\widehat{c}_{14}+2\left(\widehat{d}_{21}+\widehat{c}_{13}\right) \\
\beta_{36}=-\widehat{c}_{26}+3 \beta_{25}+\beta_{10}=-\widehat{c}_{26}+3\left(-\widehat{c}_{15}+\frac{1}{2}\left(\widehat{d}_{22}+\widehat{c}_{10}-2 \widehat{d}_{11}\right)\right)+\widehat{d}_{12} \\
\beta_{37}=-\widehat{c}_{27}+\beta_{20}=-\widehat{c}_{27}+\widehat{d}_{22} \\
\beta_{38}=-\widehat{c}_{28}+\beta_{27}+2 \beta_{26}=-\widehat{c}_{28}+\frac{1}{2} \widehat{d}_{23}+2\left(\widehat{d}_{24}\right) \\
\beta_{39}=-\widehat{c}_{29}+\beta_{28}=-\widehat{c}_{29}+\frac{1}{2} \widehat{d}_{25} \\
\beta_{30}=-\widehat{c}_{20}+\beta_{20}+2 \beta_{23}+2 \beta_{24}=-\widehat{c}_{20}+\widehat{d}_{22}+2\left(\widehat{d}_{21}\right)+2\left(-\widehat{c}_{14}+2\left(\widehat{d}_{21}+\widehat{c}_{13}\right)\right)\end{array}$ \\
\hline $\begin{array}{c}\delta_{17}=\beta_{27}+\widehat{c}_{17}-\beta_{10}=\frac{1}{2} \widehat{d}_{23}+\widehat{c}_{17}-\widehat{d}_{12} \\
\delta_{18}=\beta_{28}+\widehat{c}_{18}-2 \beta_{16}-\beta_{17}=\frac{1}{2} \widehat{d}_{25}+\widehat{c}_{18}-2 \widehat{d}_{14}-\frac{1}{2} \widehat{d}_{13} \\
\delta_{19}=\beta_{29}+\widehat{c}_{19}-\beta_{18}=\frac{1}{3} \widehat{d}_{26}+\widehat{c}_{19}-\frac{1}{2} \widehat{d}_{15}\end{array}$ \\
\hline
\end{tabular}


representing all the controls of the form (3.23), which provide, respectively, supercritical and subcritical Hopf bifurcations of the equilibrium point. Then, the corresponding bifurcation of the original system (2.7) can be turned supercritical (subcritical) without regard of the model variations, that is, it can be made supercritical (subcritical) via robust control if $r(\mu) \in R_{\text {sup }}\left(r(\mu) \in R_{\text {sub }}\right.$ ) exist, such that the corresponding $w$ does not depend on $\mu$.

Remark 3.10. When only the linear part of the starting system depends on the parameter $\mu$, any constant vector $r$ provides a robust control in the sense of the above proposition.

\section{Example}

In order to illustrate the proposed approach to Hopf bifurcation control, let us consider a system already in the form (3.3), that is,

$$
\begin{gathered}
\dot{y}_{1}=y_{2}+y_{1}^{2}-2 y_{3} y_{1}^{2}+\left(y_{1}-3 y_{3} y_{1}\right) u, \\
\dot{y}_{2}=y_{3}-2 y_{2} y_{1}-2 y_{1}^{3}+\left(y_{2}-3 y_{3} y_{2}\right) u, \\
\dot{y}_{3}=u,
\end{gathered}
$$

which corresponds to

$$
\begin{array}{cc}
f^{[2]}(y)=\left[\begin{array}{c}
y_{1}^{2} \\
-2 y_{2} y_{1} \\
0
\end{array}\right], \quad f^{[3]}(y)=\left[\begin{array}{c}
-2 y_{3} y_{1}^{2} \\
-2 y_{1}^{3} \\
0
\end{array}\right], \\
g^{[1]}(y)=\left[\begin{array}{c}
y_{1} \\
y_{2} \\
0
\end{array}\right], \quad g^{[2]}(y)=\left[\begin{array}{c}
-3 y_{3} y_{1} \\
-3 y_{3} y_{2} \\
0
\end{array}\right] .
\end{array}
$$

Then, it is straightforward to check that such a system satisfies condition (3.10), when the transformation

$$
\begin{gathered}
\varphi^{[2]}(z)=\left[\begin{array}{c}
z_{3} z_{1} \\
-z_{1}^{2}+z_{2} z_{3} \\
z_{3}^{2}
\end{array}\right], \quad \varphi^{[3]}(z)=\left[\begin{array}{c}
-z_{2} z_{1}^{2} \\
-z_{3} z_{1}^{2}-2 z_{1} z_{2}^{2} \\
-2 z_{2}^{3}-2 z_{1} z_{2} z_{3}
\end{array}\right], \\
\psi_{z}^{[2]}(z)=0, \quad \psi_{u}^{[1]}(z)=-2 z_{3}, \\
\psi_{z}^{[3]}(z)=8 z_{3} z_{2}^{2}+2 z_{1} z_{2} z_{3}, \quad \psi_{u}^{[2]}(z)=4 z_{3}^{2}-2 z_{1} z_{2}
\end{gathered}
$$


is considered. Also the following relationships hold:

$$
\hat{f}^{[3]}(z)=\left[\begin{array}{c}
0 \\
-4 z_{1} z_{2} z_{3} \\
0
\end{array}\right], \quad \hat{g}^{[2]}(z)=\left[\begin{array}{c}
0 \\
-z_{1}^{2} \\
4 z_{3}^{2}
\end{array}\right]
$$

Then, suppose that $\mu_{0}=0$, and let us assume that

$$
v_{1}(\mu)=v_{1}, \quad v_{2}(\mu)=v_{2}+\mu, \quad v_{3}(\mu)=v_{3}
$$

are such that the linear part of the system undergoes a Hopf bifurcation at $\mu=\mu_{0}$. Hence, if we consider the class of controllers (3.23) featuring

$$
r_{1}(\mu)=r_{1}, \quad r_{4}(\mu)=r_{4}, \quad r_{i}(\mu)=0, \quad i=2,3,5,6,
$$

we have that

$$
M\left(\mu_{0}\right)=\left[\begin{array}{ccccc}
-v_{3} & \frac{1}{2}\left(-r_{1}+r_{4} v_{2}\right) & 0 & 0 & 0 \\
-2 r_{1} & 0 & -r_{1}+2 r_{4} v_{2} & 0 & 2 v_{3}\left(-v_{2}\right)^{1 / 2} \\
0 & 0 & 0 & -r_{1}+2 r_{4} v_{2} & -2 v_{2} \\
0 & \frac{1}{2}\left(-r_{1}-r_{4} v_{2}\right) & 3 v_{1} & -6\left(-v_{2}\right)^{3 / 2} & 0 \\
0 & 0 & 6\left(-v_{2}\right)^{3 / 2} & 3 v_{1} & 0
\end{array}\right] .
$$

Therefore, the supercritical bifurcation condition at $\mu=\mu_{0}$ is given by

$$
\begin{aligned}
\operatorname{det} M\left(\mu_{0}\right)= & 3 v_{3}\left(r_{1}+r_{4} v_{2}\right)\left(-r_{1}+2 r_{4} v_{2}\right)\left(v_{1} v_{2}+2 v_{3}\left(-v_{2}\right)\right) \\
& -2 v_{2} r_{1}\left(r_{1}-r_{4} v_{2}\right)\left(9 v_{1}^{2}+36\left(-v_{2}\right)^{3}\right)<0
\end{aligned}
$$

defining the manifold of the pairs $\left(r_{1}, r_{4}\right)$, which realize a proper control. The explicit form of the corresponding nonlinear part of $u$ is obtained substituting the above functions and

$$
\begin{gathered}
v^{[2]}(z)=r_{1} z_{1}^{2}+r_{4} z_{2}^{2} \\
v^{[3]}(z)=0
\end{gathered}
$$

in (3.19). 


\section{Conclusions}

In this paper, we have introduced an approach to set up the Hopf bifurcation nature, without modifying the related equilibrium point features. This goal is achieved by introducing in the system specifically designed nonlinear terms, which only locally affect it in a close around of that fixed point. Then, the explicit form of classes of suitable control inputs has been obtained by exploiting local transformations, which lead the system to assume the ordinary differential equation form. In particular, we have considered third-order models, since they can be also used to represent certain classes of higher order systems. Indeed, they can be regarded as proper representations of more complex models characterized by an inner fast subsystem. Moreover, the control input has been designed via a frequency approach, which has not required the computation of the center manifold dynamics.

\section{Appendix}

\section{Proofs}

Proof of Theorem 3.1. By assumption, the pair $(\widetilde{A}(\mu), \widetilde{B}(\mu))$ is controllable for each $\mu \in \Gamma$. Therefore, it is wellknown (see, e.g. [21]) that for all $\mu \in \Gamma$, an invertible $T(\mu) \in \mathbb{R}^{3 \times 3}$ exist such that

$$
T(\mu) \tilde{A}(\mu) T^{-1}(\mu)=\left[\begin{array}{ccc}
0 & 1 & 0 \\
0 & 0 & 1 \\
v_{1}(\mu) & v_{2}(\mu) & v_{3}(\mu)
\end{array}\right], \quad T(\mu) \widetilde{B}(\mu)=\left[\begin{array}{l}
0 \\
0 \\
1
\end{array}\right] .
$$

Therefore, consider the row vector (3.1), and let us perform the linear affine transformation (20) with $T(\mu)$ defined as above. Then, it follows that

$$
\begin{aligned}
\dot{y}= & T(\mu) \tilde{A}(\mu) T^{-1}(\mu) y+T(\mu) \tilde{f}^{[2]}\left(T^{-1}(\mu) y ; \mu\right)+T(\mu) \tilde{f}^{[3]}\left(T^{-1}(\mu) y ; \mu\right) \\
& +T(\mu)\left(\widetilde{B}(\mu)+\tilde{g}^{[1]}\left(T^{-1}(\mu) y ; \mu\right)+\tilde{g}^{[2]}\left(T^{-1}(\mu) y ; \mu\right)\right) w \\
= & \left(T(\mu) \tilde{A}(\mu) T^{-1}(\mu)-T(\mu) \widetilde{B}(\mu) v(\mu)\right) y \\
& +T(\mu)\left(\tilde{f}^{[2]}\left(T^{-1}(\mu) y ; \mu\right)-\tilde{g}^{[1]}\left(T^{-1}(\mu) z ; \mu\right) v(\mu) y\right) \\
& +T(\mu)\left(\tilde{f}^{[3]}\left(T^{-1}(\mu) y ; \mu\right)-\tilde{g}^{[2]}\left(T^{-1}(\mu) y \mu\right) v(\mu) y\right) \\
& +\left(T(\mu) \widetilde{B}(\mu)+T(\mu) \tilde{g}^{[1]}\left(T^{-1}(\mu) y ; \mu\right)+T(\mu) \tilde{g}^{[2]}\left(T^{-1}(\mu) y ; \mu\right)\right)(w+v(\mu) y) \\
= & A y+f^{[2]}(y ; \mu)+f^{[3]}(y ; \mu)+\left(B+g^{[1]}(y ; \mu)+g^{[2]}(y ; \mu)\right) u,
\end{aligned}
$$

where the pair $(A, B)$ is in Brunovsky form. 
Proof of Theorem 3.3. The main idea beyond transformation (3.7) and (3.8) and representation (3.9) is derived from controller normal form theory (see [9-11, 23] for complete reference). However, hereafter we develop a slightly different approach in order to prove that model (3.9) can always be locally obtained. Moreover, thanks to the proposed approach, we are also able to point out the explicit general form of the functions (3.7) and (3.8). For the sake of simplicity, in the following, the explicit dependency on the bifurcation parameter $\mu$ will be neglected.

Hence, let us consider the nonlinear state transformation (3.7) and notice that

$$
\dot{y}=\left(I+\frac{\partial \varphi^{[2]}}{\partial z}+\frac{\partial \varphi^{[3]}}{\partial z}\right) \dot{z}
$$

which implies also that

$$
\dot{z}=\left(I+\frac{\partial \varphi^{[2]}}{\partial z}+\frac{\partial \varphi^{[3]}}{\partial z}\right)^{-1} \dot{y}
$$

Moreover, observe that

$$
\left(I+\frac{\partial \varphi^{[2]}}{\partial z}+\frac{\partial \varphi^{[3]}}{\partial z}\right)^{-1}=I-\frac{\partial \varphi^{[2]}}{\partial z}+\frac{\partial \varphi^{[2]}}{\partial z} \frac{\partial \varphi^{[2]}}{\partial z}-\frac{\partial \varphi^{[3]}}{\partial z}+O^{[3]}(z)
$$

and that

$$
\begin{gathered}
f^{[2]}\left(z+\varphi^{[2]}(z)+\cdots\right)=f^{[2]}(z)+\frac{\partial f^{[2]}}{\partial z} \varphi^{[2]}(z)+\cdots, \\
f^{[3]}(z+\cdots)=f^{[3]}(z)+\cdots, \\
g^{[1]}\left(z+\varphi^{[2]}(z)+\varphi^{[3]}(z)+\cdots\right)=g^{[1]}(z)+\frac{\partial g^{[1]}}{\partial z} \varphi^{[2]}(z)+\frac{\partial g^{[1]}}{\partial z} \varphi^{[3]}(z)+\cdots, \\
g^{[2]}\left(z+\varphi^{[2]}(z)+\cdots\right)=g^{[2]}(z)+\frac{\partial g^{[2]}}{\partial z} \varphi^{[2]}(z)+\cdots
\end{gathered}
$$


Journal of Applied Mathematics

Therefore, it results that

$$
\begin{aligned}
\dot{z}= & \left(I-\frac{\partial \varphi^{[2]}}{\partial z}+\frac{\partial \varphi^{[2]}}{\partial z} \frac{\partial \varphi^{[2]}}{\partial z}-\frac{\partial \varphi^{[3]}}{\partial z}+O^{[3]}(z)\right) \\
& \times\left(A z+A \varphi^{[2]}(z)+A \varphi^{[3]}(z)+f^{[2]}(z)+\frac{\partial f^{[2]}}{\partial z} \varphi^{[2]}(z)+f^{[3]}(z)+O^{[4]}(z)\right. \\
& \left.+\left(B+g^{[1]}(z)+\frac{\partial g^{[1]}}{\partial z} \varphi^{[2]}(z)+g^{[2]}(z)+O^{[2]}(z)\right) u\right) \\
= & A y+B u \\
& +A \varphi^{[2]}(z)+f^{[2]}(z)-\frac{\partial \varphi^{[2]}}{\partial z} A z-\frac{\partial \varphi^{[2]}}{\partial z} B u+g^{[1]}(z) u \\
& +A \varphi^{[3]}(z)+\frac{\partial f^{[2]}}{\partial z} \varphi^{[2]}(z)+f^{[3]}(z)+\frac{\partial g^{[1]}}{\partial z} \varphi^{[2]}(z) u+g^{[2]}(z) u \\
& -\frac{\partial \varphi^{[2]}}{\partial z} A \varphi^{[2]}(z)-\frac{\partial \varphi^{[2]}}{\partial z} f^{[2]}(z)-\frac{\partial \varphi^{[2]}}{\partial z} g^{[1]}(z) u \\
& +\frac{\partial \varphi^{[2]}}{\partial z} \frac{\partial \varphi^{[2]}}{\partial z} A z-\frac{\partial \varphi^{[3]}}{\partial z} A z+\frac{\partial \varphi^{[2]}}{\partial z} \frac{\partial \varphi^{[2]}}{\partial z} B u-\frac{\partial \varphi^{[3]}}{\partial z} B u+\cdots
\end{aligned}
$$

Let us first study the quadratic terms (A.8) of the above representation. According to (3.9) and (3.8), it must result that

$$
\begin{gathered}
\varphi_{2}^{[2]}(z)+f_{1}^{[2]}(z)-\frac{\partial \varphi_{1}^{[2]}}{\partial z_{1}} z_{2}-\frac{\partial \varphi_{1}^{[2]}}{\partial z_{2}} z_{3}-\frac{\partial \varphi_{1}^{[2]}}{\partial z_{3}} u+g_{1}^{[1]}(z) u=\gamma_{16} z_{3}^{2} \\
\varphi_{3}^{[2]}(z)+f_{2}^{[2]}(z)-\frac{\partial \varphi_{2}^{[2]}}{\partial z_{1}} z_{2}-\frac{\partial \varphi_{2}^{[2]}}{\partial z_{2}} z_{3}-\frac{\partial \varphi_{2}^{[2]}}{\partial z_{3}} u+g_{2}^{[1]}(z) u=0 \\
f_{3}^{[2]}(z)-\frac{\partial \varphi_{3}^{[2]}}{\partial z_{1}} z_{2}-\frac{\partial \varphi_{3}^{[2]}}{\partial z_{2}} z_{3}-\frac{\partial \varphi_{3}^{[2]}}{\partial z_{3}} u+g_{3}^{[1]}(z) u=\psi^{[2]}(z, u) .
\end{gathered}
$$

Let us denote

$$
\begin{gathered}
f_{i}^{[2]}(z)=a_{i 1} z_{1}^{2}+a_{i 2} z_{2} z_{1}+a_{i 3} z_{3} z_{1}+a_{i 4} z_{2}^{2}+a_{i 5} z_{3} z_{2}+a_{i 6} z_{3}^{2}, \\
g_{i}^{[1]}(z)=b_{i 1} z_{1}+b_{i 2} z_{2}+b_{i 3} z_{3}, \\
\varphi_{i}^{[2]}(z)=\alpha_{i 1} z_{1}^{2}+\alpha_{i 2} z_{2} z_{1}+\alpha_{i 3} z_{3} z_{1}+\alpha_{i 4} z_{2}^{2}+\alpha_{i 5} z_{3} z_{2}+\alpha_{i 6} z_{3}^{2}, \quad i=1,2,3 .
\end{gathered}
$$


Then, in order to delete $u$ form the first and second equations, the following parametric conditions have to be satisfied:

$$
\frac{\partial \varphi_{i}^{[2]}}{\partial z_{3}}=\alpha_{i 3} z_{1}+\alpha_{i 5} z_{2}+2 \alpha_{i 6} z_{3}=b_{i 1} z_{1}+b_{i 2} z_{2}+b_{i 3} z_{3}, \quad i=1,2
$$

thus, leading to the relationships

$$
\alpha_{i 3}=b_{i 1}, \quad \alpha_{i 5}=b_{i 2}, \quad \alpha_{i 6}=\frac{1}{2} b_{i 3}, \quad i=1,2
$$

Then, by observing that

$$
\begin{aligned}
& \frac{\partial \varphi_{1}^{[2]}}{\partial z_{2}} z_{3}=\alpha_{12} z_{3} z_{1}+2 \alpha_{14} z_{3} z_{2}+\alpha_{15} z_{3}^{2}=\alpha_{12} z_{3} z_{1}+2 \alpha_{14} z_{3} z_{2}+b_{12} z_{3}^{2} \\
& \frac{\partial \varphi_{1}^{[2]}}{\partial z_{1}} z_{2}=2 \alpha_{11} z_{2} z_{1}+\alpha_{12} z_{2}^{2}+\alpha_{13} z_{3} z_{2}=2 \alpha_{11} z_{2} z_{1}+\alpha_{12} z_{2}^{2}+b_{11} z_{3} z_{2}
\end{aligned}
$$

the following considerations are in order. After deleting $u$ in the second equation (A.11), the other components of $\varphi_{2}^{[2]}(z)$ could be used to delete from (A.10) the terms $z_{1}^{2}, z_{1} z_{2}$ and $z_{2}^{2}$. Similarly, after deleting $u$ in (A.10) by means of $\varphi_{1}^{[2]}(z)$, this function could also be employed to delete $z_{1} z_{2}, z_{2} z_{3}$, and one between $z_{1} z_{3}$, and $z_{2}^{2}$. The optimal choice in order to delete most of the elements is taking care of $z_{2} z_{3}$ and $z_{1} z_{3}$. Doing that way, it is straightforward to check that the only quadratic term of the first equation (A.10) that cannot be deleted by a proper choice of $\varphi^{[2]}(z)$ is $z_{3}^{2}$.

The rest of the second equation (A.11), conversely, can be delete by choosing

$$
\varphi_{3}^{[2]}(z)=-f_{2}^{[2]}(z)+\frac{\partial \varphi_{2}^{[2]}}{\partial z_{1}} z_{2}+\frac{\partial \varphi_{2}^{[2]}}{\partial z_{2}} z_{3}
$$

while third equation (A.12) just becomes the very definition of $\psi^{[2]}(z, u)$. The related coefficients of $\varphi^{[2]}(z)$ are reported in Table 1 and in particular it results that

$$
\gamma_{16}=\alpha_{26}+a_{16}-b_{12}=\frac{1}{2} b_{23}+a_{16}-b_{12} .
$$

Let us now consider the cubic part of the problem. First, let us denote

$$
\begin{gathered}
\hat{f}^{[3]}(z)=f^{[3]}(z)+\frac{\partial f^{[2]}}{\partial z} \varphi^{[2]}(z)-\frac{\partial \varphi^{[2]}}{\partial z} A \varphi^{[2]}(z)-\frac{\partial \varphi^{[2]}}{\partial z} f^{[2]}(z)+\frac{\partial \varphi^{[2]}}{\partial z} \frac{\partial \varphi^{[2]}}{\partial z} A z \\
\widehat{g}^{[2]}(z)=g^{[2]}(z)+\frac{\partial g^{[1]}}{\partial z} \varphi^{[2]}(z)-\frac{\partial \varphi^{[2]}}{\partial z} g^{[1]}(z)+\frac{\partial \varphi^{[2]}}{\partial z} \frac{\partial \varphi^{[2]}}{\partial z} B
\end{gathered}
$$


and for $i=1,2,3$,

$$
\begin{aligned}
f_{i}^{[3]}(z)= & c_{i 1} z_{1}^{3}+c_{i 2} z_{2} z_{1}^{2}+c_{i 3} z_{3} z_{1}^{2}+c_{i 4} z_{1} z_{2}^{2}+c_{i 5} z_{2}^{3}+c_{i 6} z_{3} z_{2}^{2} \\
& +c_{i 7} z_{1} z_{3}^{2}+c_{i 8} z_{2} z_{3}^{2}+c_{i 9} z_{3}^{3}+c_{i 0} z_{1} z_{2} z_{3}, \\
g_{i}^{[2]}(z)= & d_{i 1} z_{1}^{2}+d_{i 2} z_{2} z_{1}+d_{i 3} z_{3} z_{1}+d_{i 4} z_{2}^{2}+d_{i 5} z_{3} z_{2}+d_{i 6} z_{3}^{2}, \\
\widehat{f}_{i}^{[3]}(z)= & \widehat{c}_{i 1} z_{1}^{3}+\widehat{c}_{i 2} z_{2} z_{1}^{2}+\widehat{c}_{i 3} z_{3} z_{1}^{2}+\widehat{c}_{i 4} z_{1} z_{2}^{2}+\widehat{c}_{i 5} z_{2}^{3}+\widehat{c}_{i 6} z_{3} z_{2}^{2} \\
& +\widehat{c}_{i 7} z_{1} z_{3}^{2}+\widehat{c}_{i 8} z_{2} z_{3}^{2}+\widehat{c}_{i 9} z_{3}^{3}+\widehat{c}_{i 0} z_{1} z_{2} z_{3}, \\
\widehat{g}_{i}^{[2]}(z)= & \widehat{d}_{i 1} z_{1}^{2}+\widehat{d}_{i 2} z_{2} z_{1}+\widehat{d}_{i 3} z_{3} z_{1}+\widehat{d}_{i 4} z_{2}^{2}+\widehat{d}_{i 5} z_{3} z_{2}+\widehat{d}_{i 6} z_{3}^{2}, \\
\varphi_{i}^{[3]}(z)= & \beta_{i 1} z_{1}^{3}+\beta_{i 2} z_{2} z_{1}^{2}+\beta_{i 3} z_{3} z_{1}^{2}+\beta_{i 4} z_{1} z_{2}^{2}+\beta_{i 5} z_{2}^{3}+\beta_{i 6} z_{3} z_{2}^{2}+\beta_{i 7} z_{1} z_{3}^{2} \\
& +\beta_{i 8} z_{2} z_{3}^{2}+\beta_{i 9} z_{3}^{3}+\beta_{i 0} z_{1} z_{2} z_{3} .
\end{aligned}
$$

Then, by observing for each $i=1,2,3$ that

$$
\begin{aligned}
\hat{f}_{i}^{[3]}(z)= & f_{i}^{[3]}(z)+\frac{\partial f_{i}^{[2]}}{\partial z_{1}} \varphi_{1}^{[2]}(z)+\frac{\partial f_{i}^{[2]}}{\partial z_{2}} \varphi_{2}^{[2]}(z)+\frac{\partial f_{i}^{[2]}}{\partial z_{3}} \varphi_{3}^{[2]}(z) \\
& -\frac{\partial \varphi_{i}^{[2]}}{\partial z_{1}} \varphi_{2}^{[2]}(z)-\frac{\partial \varphi_{i}^{[2]}}{\partial z_{2}} \varphi_{3}^{[2]}(z) \\
& -\frac{\partial \varphi_{i}^{[2]}}{\partial z_{1}} f_{1}^{[2]}(z)-\frac{\partial \varphi_{i}^{[2]}}{\partial z_{2}} f_{2}^{[2]}(z)-\frac{\partial \varphi_{i}^{[2]}}{\partial z_{3}} f_{3}^{[2]}(z) \\
& +\frac{\partial \varphi_{i}^{[2]}}{\partial z_{1}} \frac{\partial \varphi_{1}^{[2]}}{\partial z_{1}} z_{2}+\frac{\partial \varphi_{i}^{[2]}}{\partial z_{1}} \frac{\partial \varphi_{1}^{[2]}}{\partial z_{2}} z_{3}+\frac{\partial \varphi_{i}^{[2]}}{\partial z_{2}} \frac{\partial \varphi_{2}^{[2]}}{\partial z_{1}} z_{2} \\
& +\frac{\partial \varphi_{i}^{[2]}}{\partial z_{2}} \frac{\partial \varphi_{2}^{[2]}}{\partial z_{2}} z_{3}+\frac{\partial \varphi_{i}^{[2]}}{\partial z_{3}} \frac{\partial \varphi_{3}^{[2]}}{\partial z_{1}} z_{2}+\frac{\partial \varphi_{i}^{[2]}}{\partial z_{3}} \frac{\partial \varphi_{3}^{[2]}}{\partial z_{2}} z_{3}, \\
\hat{g}_{i}^{[2]}(z)= & g_{i}^{[2]}(z)+\frac{\partial g_{i}^{[1]}}{\partial z_{1}} \varphi_{1}^{[2]}(z)+\frac{\partial g_{i}^{[1]}}{\partial z_{2}} \varphi_{2}^{[2]}(z)+\frac{\partial g_{i}^{[1]}}{\partial z_{3}} \varphi_{3}^{[2]}(z) \\
& -\frac{\partial \varphi_{i}^{[2]}}{\partial z} g_{1}^{[1]}(z)-\frac{\partial \varphi_{i}^{[2]}}{\partial z} g_{2}^{[1]}(z)-\frac{\partial \varphi_{i}^{[2]}}{\partial z} g_{3}^{[1]}(z) \\
& +\frac{\partial \varphi_{i}^{[2]}}{\partial z_{1}} \frac{\partial \varphi_{1}^{[2]}}{\partial z_{3}}+\frac{\partial \varphi_{i}^{[2]}}{\partial z_{2}} \frac{\partial \varphi_{2}^{[2]}}{\partial z_{3}}+\frac{\partial \varphi_{i}^{[2]}}{\partial z_{3}} \frac{\partial \varphi_{3}^{[2]}}{\partial z_{3}}
\end{aligned}
$$

the relationships among the coefficients of the pair $f^{[3]}(z), g^{[2]}(z, u)$ and those of $\tilde{f}^{[3]}(z)$, $\tilde{g}^{[2]}(z, u)$ turn out to be as reported in Tables 2 and 3 . 

the form

Exploiting the above results, let us consider the cubic part problem (71). It assumes

$$
\begin{gathered}
A \varphi^{[3]}(z)+\widehat{f}^{[3]}(z)-\frac{\partial \varphi^{[3]}}{\partial z} A z-\frac{\partial \varphi^{[3]}}{\partial z} B u+\hat{g}^{[2]}(z) u \\
=\left[\delta_{17} z_{3}^{3}+\delta_{18} z_{1} z_{3}^{2}+\delta_{19} z_{2} z_{3}^{2} \quad 0 \quad \psi^{[3]}(\mathrm{z}, u)\right]^{T},
\end{gathered}
$$

that is,

$$
\begin{gathered}
\varphi_{2}^{[3]}(z)+\widehat{f}_{1}^{[3]}(z)-\frac{\partial \varphi_{1}^{[3]}}{\partial z_{1}} z_{2}-\frac{\partial \varphi_{1}^{[3]}}{\partial z_{2}} z_{3}-\frac{\partial \varphi_{1}^{[3]}}{\partial z_{3}} u+\widehat{g}_{1}^{[2]}(z) u=\delta_{17} z_{3}^{3}+\delta_{18} z_{1} z_{3}^{2}+\delta_{19} z_{2} z_{3}^{2} \\
\varphi_{3}^{[3]}(z)+\widehat{f}_{2}^{[3]}(z)-\frac{\partial \varphi_{2}^{[3]}}{\partial z_{1}} z_{2}-\frac{\partial \varphi_{2}^{[3]}}{\partial z_{2}} z_{3}-\frac{\partial \varphi_{2}^{[3]}}{\partial z_{3}} u+\widehat{g}_{2}^{[2]}(z) u=0 \\
\hat{f}_{3}^{[3]}(z)-\frac{\partial \varphi_{3}^{[3]}}{\partial z_{1}} z_{2}-\frac{\partial \varphi_{3}^{[3]}}{\partial z_{2}} z_{3}-\frac{\partial \varphi_{3}^{[3]}}{\partial z_{3}} u+\widehat{g}_{3}^{[2]}(z) u=\psi^{[3]}(z, u) .
\end{gathered}
$$

Then, we may proceed similarly to the quadratic case. Deleting $u$ from the first and second equations (A.24) and (A.25) implies that

$$
\begin{aligned}
\frac{\partial \varphi_{i}^{[3]}}{\partial z_{3}} & =\beta_{i 3} z_{1}^{2}+\beta_{i 6} z_{2}^{2}+2 \beta_{i 7} z_{1} z_{3}+2 \beta_{i 8} z_{2} z_{3}+3 \beta_{i 9} z_{3}^{2}+\beta_{i 0} z_{1} z_{2} \\
& =\widehat{d}_{i 1} z_{1}^{2}+\widehat{d}_{i 2} z_{2} z_{1}+\widehat{d}_{i 3} z_{3} z_{1}+\widehat{d}_{i 4} z_{2}^{2}+\widehat{d}_{i 5} z_{3} z_{2}+\widehat{d}_{i 6} z_{3}^{2}, \quad i=1,2,
\end{aligned}
$$

which corresponds to the following parametric conditions:

$$
\begin{gathered}
\beta_{i 3}=\widehat{d}_{i 1}, \quad \beta_{i 6}=\widehat{d}_{i 4}, \quad \beta_{i 7}=\frac{1}{2} \widehat{d}_{i 3}, \\
\beta_{i 8}=\frac{1}{2} \widehat{d}_{i 5}, \quad \beta_{i 9}=\frac{1}{3} \widehat{d}_{i 6}, \quad \beta_{i 0}=\widehat{d}_{i 2}, \quad i=1,2 .
\end{gathered}
$$

Therefore, by observing that

$$
\begin{aligned}
& \frac{\partial \varphi_{1}^{[3]}}{\partial z_{2}} z_{3}=\beta_{12} z_{1}^{2} z_{3}+2 \beta_{14} z_{1} z_{2} z_{3}+3 \beta_{15} z_{2}^{2} z_{3}+2 \beta_{16} z_{2} z_{3}^{2}+\beta_{18} z_{3}^{3}+\beta_{10} z_{1} z_{3}^{2} \\
& \frac{\partial \varphi_{1}^{[3]}}{\partial z_{1}} z_{2}=3 \beta_{11} z_{1}^{2} z_{2}+2 \beta_{12} z_{1} z_{2}^{2}+2 \beta_{13} z_{1} z_{3} z_{2}+\beta_{14} z_{2}^{3}+\beta_{17} z_{3}^{2} z_{2}+\beta_{10} z_{3} z_{2}^{2}
\end{aligned}
$$

it is straightforward to check that $\varphi_{2}^{[3]}(z)$ still allows one to delete in the first equation (A.24) the terms $z_{1}^{3}, z_{2} z_{1}^{2}, z_{1} z_{2}^{2}$, and $z_{2}^{3}$. By exploiting $\varphi_{1}^{[3]}(z)$, in turn, one could delete in the same equation also the terms $z_{3} z_{2}^{2}$ (via $\left.\beta_{15}\right), z_{2} z_{1}^{2}$ (via $\beta_{11}$ ), one between $z_{3} z_{1}^{2}$ and $z_{1} z_{2}^{2}$ (via $\left.\beta_{12}\right)$ and 
one between $z_{1} z_{2} z_{3}$ and $z_{2}^{3}$ (via $\beta_{14}$ ). Hence, by choosing to delete via $\varphi_{1}^{[3]}(z)$ the terms $z_{3} z_{2}^{2}$, $z_{3} z_{1}^{2}$ and $z_{1} z_{2} z_{3}$, we have that in the first equation (A.24) only the terms $z_{3}^{3}, z_{1} z_{3}^{2}$, and $z_{2} z_{3}^{2}$ still remain. Then, in order to delete every one element in the second equation (A.25), we set

$$
\varphi_{3}^{[3]}(z)=-\widehat{f}_{2}^{[3]}(z)+\frac{\partial \varphi_{2}^{[3]}}{\partial z_{1}} z_{2}+\frac{\partial \varphi_{2}^{[3]}}{\partial z_{2}} z_{3}
$$

Finally, the third equation (A.26) can be regarded just as the definition of $\psi^{[3]}(z, u)$. The parametric relationships related to $\varphi^{[3]}(z)$ and $\psi^{[3]}(z, u)$ are reported in Table 4 . In particular, we have that

$$
\begin{gathered}
\delta_{17}=\beta_{27}+\widehat{c}_{17}+\beta_{10}, \\
\delta_{18}=\beta_{28}+\widehat{c}_{18}+2 \beta_{16}+\beta_{17}, \\
\delta_{19}=\beta_{29}+\widehat{c}_{19}+\beta_{18} .
\end{gathered}
$$

Proof of Theorem 3.5. Consider system (A.10)-(A.12), which describe the transformation equation for the quadratic component (A.8). Moreover, denote

$$
\begin{aligned}
& \psi^{[2]}(z, u)=\psi_{z}^{[2]}(z)+\psi_{u}^{[1]}(z) u, \\
& \psi^{[3]}(z, u)=\psi_{z}^{[3]}(z)+\psi_{u}^{[2]}(z) u .
\end{aligned}
$$

Then, it is straightforward to check that balancing (A.10)-(A.12) under condition (3.10) is equivalent to solving (3.13) and (3.14).

Observe that

$$
\begin{aligned}
\widehat{f}^{[3]}(z) & =f^{[3]}(z)+\frac{\partial f^{[2]}}{\partial z} \varphi^{[2]}(z)-\frac{\partial \varphi^{[2]}}{\partial z}\left(A \varphi^{[2]}(z)+f^{[2]}(z)-\frac{\partial \varphi^{[2]}}{\partial z} A z\right) \\
& =f^{[3]}(z)+\frac{\partial f^{[2]}}{\partial z} \varphi^{[2]}(z)-\frac{\partial \varphi^{[2]}}{\partial z} B \psi_{z}^{[2]}(z), \\
\hat{g}^{[2]}(z) & =g^{[2]}(z)+\frac{\partial g^{[1]}}{\partial z} \varphi^{[2]}(z)-\frac{\partial \varphi^{[2]}}{\partial z}\left(g^{[1]}(z)-\frac{\partial \varphi^{[2]}}{\partial z} B\right) \\
& =g^{[2]}(z)+\frac{\partial g^{[1]}}{\partial z} \varphi^{[2]}(z)-\frac{\partial \varphi^{[2]}}{\partial z} B \varphi_{u}^{[1]}(z) .
\end{aligned}
$$

Then, consider system (A.24)-(A.26), related to the constraints on (71). Exploiting the above functions in the computation of (71), it follows that solving (A.24)-(A.26) under condition (3.10) boils down to find the solutions of just (3.15) and (3.16). 
Proof of Theorem 3.6. Let us consider control (3.17) and the local nonlinear state change of coordinate (3.7). Moreover, observe that the nonlinear terms of (3.17) in the new variable $z$ satisfy

$$
\begin{gathered}
k^{[2]}\left(z+\varphi^{[2]}(z)+\cdots ; \mu\right)=k^{[2]}(z ; \mu)+\frac{\partial k^{[2]}}{\partial z} \varphi^{[2]}(z ; \mu)+\cdots, \\
k^{[3]}(z+\cdots ; \mu)=k^{[3]}(z ; \mu)+\cdots .
\end{gathered}
$$

Hence, we have that such an input can be locally written as a feedback of the state $z$ according to the following representation:

$$
u=v(\mu) z+v(\mu) \varphi^{[2]}(z)+v(\mu) \varphi^{[3]}(z)+k^{[2]}(z ; \mu)+\frac{\partial k^{[2]}}{\partial z} \varphi^{[2]}(z ; \mu)+k^{[3]}(z ; \mu) .
$$

Notice that we have neglected the higher terms, since we are dealing with local equivalence (i.e., up to the third-order elements).

Then, exploiting the above $u$ in the transformation (A.35) and observing that

$$
\begin{aligned}
& \psi^{[2]}\left(z, v(\mu) z+v(\mu) \varphi^{[2]}(z)+k^{[2]}(z ; \mu)+\cdots\right) \\
& \quad=\psi^{[2]}(z, v(\mu) z)+\frac{\partial \psi^{[2]}}{\partial u} \varphi^{[2]}(z)+\frac{\partial \psi^{[2]}}{\partial u} k^{[2]}(z ; \mu)+\cdots, \\
& \psi^{[3]}(z, v(\mu) z+\cdots)=\psi^{[3]}(z, v(\mu) z)+\cdots,
\end{aligned}
$$

we can compute the locally equivalent input (3.18), expressed as a function of $z$, via the following equation:

$$
\begin{aligned}
v= & v^{[1]}(z)+v^{[2]}(z)+v^{[3]}(z) \\
= & v(\mu) z+v(\mu) \varphi^{[2]}(z)+v(\mu) \varphi^{[3]}(z)+k^{[2]}(z ; \mu)+\frac{\partial k^{[2]}}{\partial z} \varphi^{[2]}(z ; \mu)+k^{[3]}(z ; \mu) \\
& +\psi^{[2]}(z, v(\mu) z)+\frac{\partial \psi^{[2]}}{\partial u} \varphi^{[2]}(z)+\frac{\partial \psi^{[2]}}{\partial u} k^{[2]}(z ; \mu)+\psi^{[3]}(z, v(\mu) z) .
\end{aligned}
$$

\section{Tables}

(The proposed approach is based on multiple changes of the state variables and the input command. It is important to notice that the global and local transformations illustrated above can always be described explicitly by deriving the relationships between the coefficients of the involved functions. Therefore, such transformations have to be computed only once, then using the resulting parametric dependencies as look-up tables. In this regard, we report these explicit relationships in Tables 1-4). 


\section{References}

[1] E. H. Abed, H. O. Wang, and A. Tesi, "Control of bifurcation and chaos," in The Control Handbook, chapter 57.6, pp. 951-966, CRC Press, Boca Raton, Fla, USA, 1996.

[2] G. Chen, J. L. Moiola, and H. O. Wang, "Bifurcation control: theories, methods, and applications," International Journal of Bifurcation and Chaos in Applied Sciences and Engineering, vol. 10, no. 3, pp. 511$548,2000$.

[3] H.-C. Lee and E. H. Abed, "Washout filters in the bifurcation control of high alpha flight dynamics," in Proceedings of the American Control Conference, pp. 206-211, Boston, Mass, USA, 1991.

[4] H. O. Wang and E. H. Abed, "Bifurcation control of a chaotic system," Automatica, vol. 31, no. 9, pp. 1213-1226, 1995.

[5] E. H. Abed and J.-H. Fu, "Local feedback stabilization and bifurcation control-I. Hopf bifurcation," Systems \& Control Letters, vol. 7, no. 1, pp. 11-17, 1986.

[6] E. H. Abed and J.-H. Fu, "Local feedback stabilization and bifurcation control-II. Stationary bifurcation," Systems \& Control Letters, vol. 8, no. 5, pp. 467-473, 1987.

[7] P. Yu and G. Chen, "Hopf bifurcation control using nonlinear feedback with polynomial functions," International Journal of Bifurcation and Chaos in Applied Sciences and Engineering, vol. 14, no. 5, pp. 16831704, 2004.

[8] B. Hamzi, W. Kang, and J.-P. Barbot, "Analysis and control of Hopf bifurcations," SIAM Journal on Control and Optimization, vol. 42, no. 6, pp. 2200-2220, 2004.

[9] W. Kang, "Bifurcation and normal form of nonlinear control systems-I," SIAM Journal on Control and Optimization, vol. 36, no. 1, pp. 193-212, 1998.

[10] W. Kang, "Bifurcation and normal form of nonlinear control systems-II," SIAM Journal on Control and Optimization, vol. 36, no. 1, pp. 213-232, 1998.

[11] W. Kang, "Bifurcation control via state feedback for systems with a single uncontrollable mode," SIAM Journal on Control and Optimization, vol. 38, no. 5, pp. 1428-1452, 2000.

[12] F. Verduzco and J. Alvarez, "Hopf bifurcation control: a new approach," Systems E Control Letters, vol. 55, no. 6, pp. 437-451, 2006.

[13] D. W. Berns, J. L. Moiola, and G. Chen, "Feedback control of limit cycle amplitudes from a frequency domain approach," Automatica, vol. 34, no. 12, pp. 1567-1573, 1998.

[14] J. L. Moiola and G. Chen, Hopf Bifurcation Analysis, vol. 21, World Scientific Publishing, River Edge, NJ, USA, 1996.

[15] A. Tesi, E. H. Abed, R. Genesio, and H. O. Wang, "Harmonic balance analysis of period-doubling bifurcations with implications for control of nonlinear dynamics," Automatica, vol. 32, no. 9, pp. 12551271, 1996.

[16] G. Innocenti, R. Genesio, and C. Ghilardi, "Oscillations and chaos in simple quadratic systems," International Journal of Bifurcation and Chaos, vol. 18, no. 7, pp. 1917-1937, 2008.

[17] D. J. Allwright, "Harmonic balance and the Hopf bifurcation," Mathematical Proceedings of the Cambridge Philosophical Society, vol. 82, no. 3, pp. 453-467, 1977.

[18] A. I. Mees, Dynamics of Feedback Systems, John Wiley \& Sons, Chichester, UK, 1981.

[19] G. Innocenti, A. Tesi, and R. Genesio, "Complex behavior analysis in quadratic jerk systems via frequency domain hopf bifurcation," International Journal of Bifurcation and Chaos, vol. 20, no. 3, pp. 657-667, 2010.

[20] G. Innocenti, Analysis and control of the Hopf bifurcation, Ph.D. thesis, Univerisità di Firenze, 2008.

[21] H. K. Khalil, Nonlinear Systems, Prentice Hall, Singapore, 2001.

[22] G. Innocenti, A. Tesi, and R. Genesio, "Hopf bifurcations in normal forms of third order nonlinear affine control systems," in Proceedings of the 17th IFAC World Congress, vol. 17, pp. 15119-15124, Seoul, Republic of Korea, 2008.

[23] W. Kang and A. J. Krener, "Extended quadratic controller normal form and dynamic state feedback linearization of nonlinear systems," SIAM Journal on Control and Optimization, vol. 30, no. 6, pp. 13191337, 1992. 


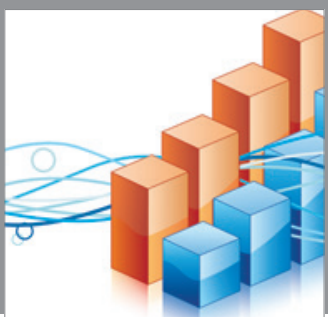

Advances in

Operations Research

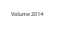

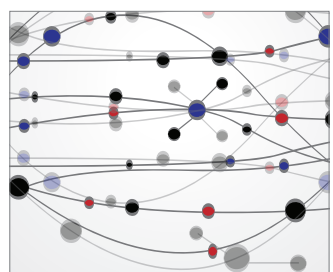

\section{The Scientific} World Journal
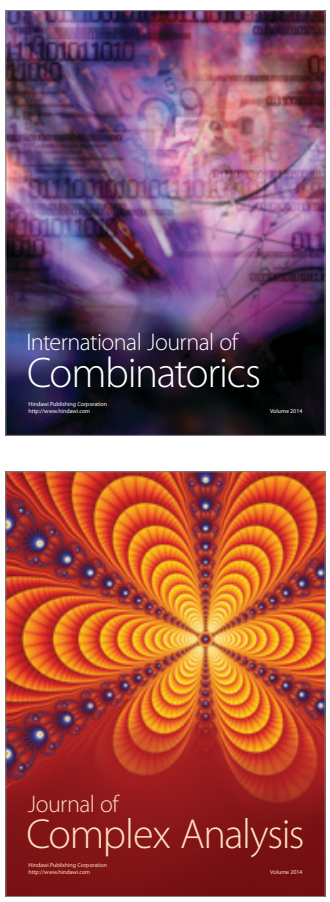

International Journal of

Mathematics and

Mathematical

Sciences
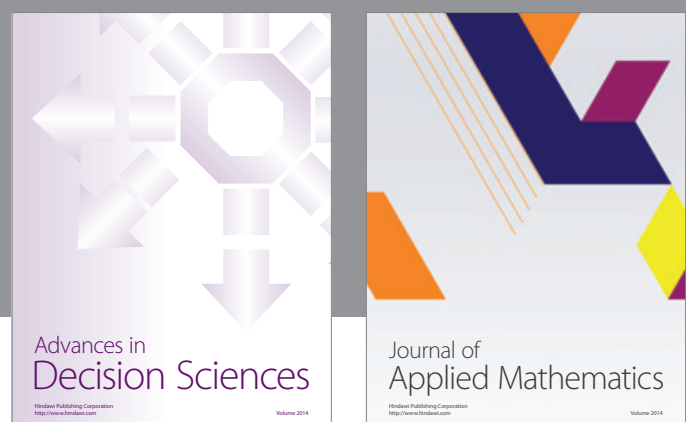

Journal of

Applied Mathematics
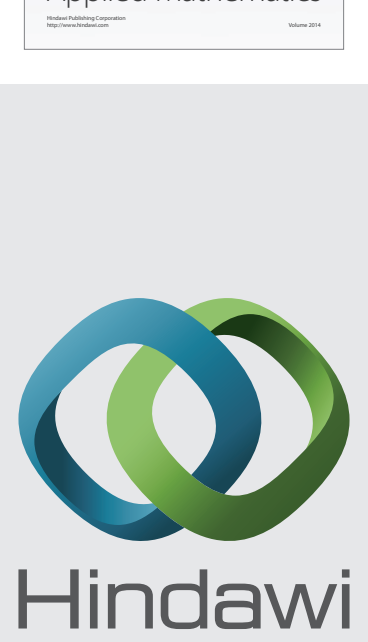

Submit your manuscripts at http://www.hindawi.com
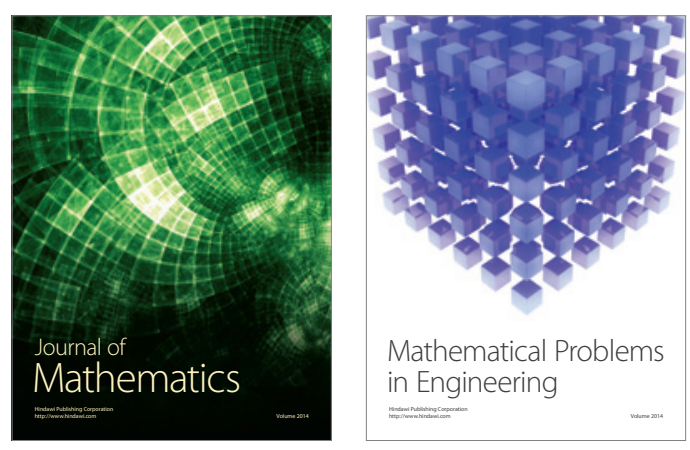

Mathematical Problems in Engineering
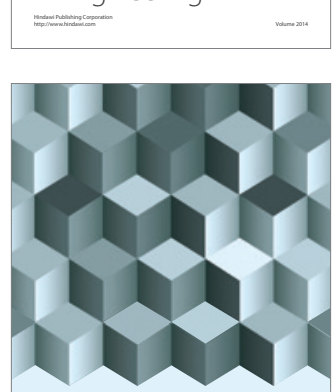

Journal of

Function Spaces
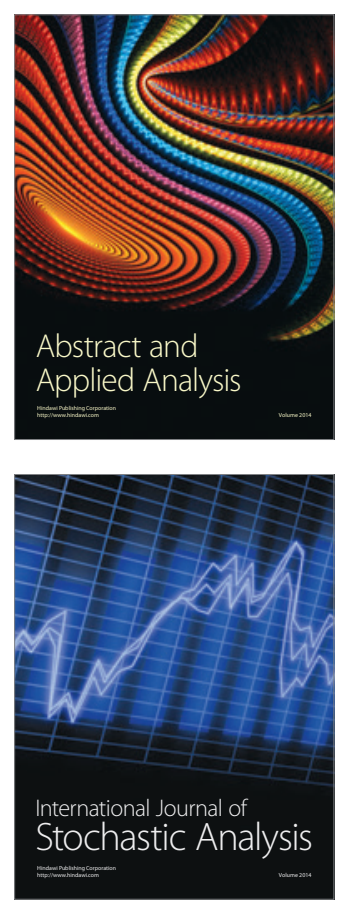

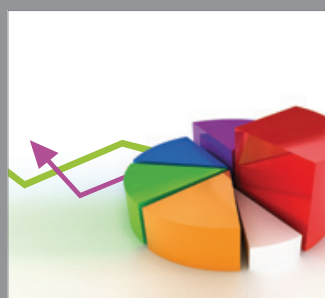

ournal of

Probability and Statistics

Promensencen
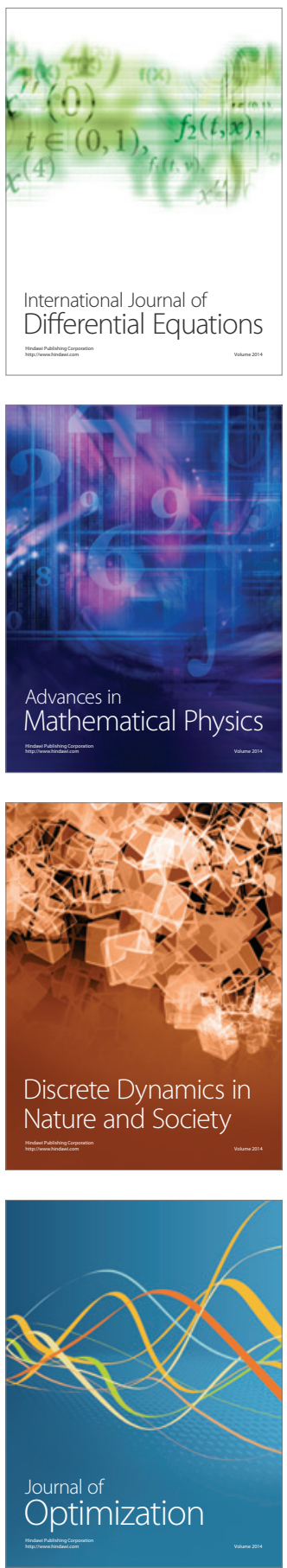